\title{
Revisiting an Outreach Mathematician
}

\section{Jerry Dwyer and Lawrence Schovanec}

Two articles describing early experiences of an outreach mathematician and the chairperson who advocated for such a role appeared in the Notices of the American Mathematical Society in 2001 [1], [2]. Several years later, it is timely to reflect again on the evolving nature of this endeavor. The first author (Dwyer) of this essay is the outreach mathematician involved in the earlier articles. The second author (Schovanec) was the chair of the Department of Mathematics and Statistics (M\&S) at Texas Tech University (TTU) at the time that Dwyer was hired in 2003. Schovanec now serves as the interim president of TTU and previously was the dean of the College of Arts \& Sciences. In each of his administrative roles he has promoted outreach, engagement, and the associated reward structures within the university.

\section{Background}

Herein we define outreach for a mathematics department as any activity that enhances the teaching and learning of mathematics outside the department, in particular in K-12 education and community colleges. An outreach mathematician is not a mathematics education researcher but is likely to spend considerable time working on matters that are sometimes within the domain of mathematics education. A significant difference

Jerry Dwyer is professor of mathematics at Texas Tech University. His email address is jerry.dwyer@ttu . edu.

Lawrence Schovanec is interim president at Texas Tech University. His email address is 1awrence.schovanec@ ttu. edu.

Members of the Editorial Board for Doceamus are: David Bressoud, Roger Howe, Karen King, William McCallum, and Mark Saul.

DOI: http://dx.doi.org/10.1090/noti1023 between outreach and traditional mathematics scholarship is dependence on the cooperation of local school districts and state educational agencies and of teachers at all levels.

Reaction to the 2001 articles was positive. Many institutions have faculty dedicated to outreach, but in many cases these faculty were hired in nontenuretrack roles or pursued outreach activities only when the traditional requirements for tenure and promotion were met. This is because the typical reward system may not recognize that an outreach mathematician should be tenured and promoted in a manner cognizant of his or her outreach role as is the case in a traditional track of pure mathematical or educational research. Addressing this issue is critical to any effort to create an outreach position in a mathematics department.

At the time of the earlier articles, Dwyer was a tenure-track faculty member in the Department of Mathematics at the University of Tennessee. In spite of satisfaction with the job and a high level of support from the Tennessee mathematics faculty, for personal reasons, he moved to the M\&S department at TTU in 2003. When this position became vacant in 2002, there was mixed support to add faculty expertise in mathematics education, but Schovanec, then the department chair, recognized the potential of adding an outreach faculty member. Moving to a new position had its challenges, including building confidence with the local K-12 community, obtaining support of new colleagues, and a delay in the tenure and promotion process for the outreach mathematician.

\section{Outreach Roles and Recognition}

At TTU, there are basically three components in the outreach work: (1) on-campus activity teaching courses, supervising students, and interacting 
with faculty in other departments who have interests that are aligned with outreach activities; (2) organizing locally and regionally funded outreach activities such as K-12 school visits and summer programs; and (3) serving on national committees and participating on panels at various meetings. There has been a progression in how these roles have been developed. Master's theses have been completed and a mathematics education concentration has been added to the mathematics Ph.D. program that provides opportunities for training mathematicians in the newer outreach roles. Personal school visits have been reduced, while teacher workshops and collaboration with school districts have increased. Grant writing has evolved. Though proposals are still submitted to local foundations with a history of supporting educational programs unique to TTU, a large commitment is now tied to substantial federal funding requests, often of a collaborative nature.

The major issues facing an outreach mathematician are similar to those of traditional faculty. There is a need to seek funding and to publish scholarly articles. A progression from small funded proposals to larger ones has resulted in significant support for outreach projects. Some of the activities for which funding has been obtained include girls' math clubs, pre-engineering and mathematics academy summer programs, and several National Science Foundation programs primarily related to teacher training and scholarship programs. Common objectives of these programs include: increasing the number and diversity of students in STEM programs; enhancing undergraduate research opportunities in the sciences; and growing the number of teachers in STEM areas while enhancing opportunities for teacher preparation.

One award, which is reflective of the maturation of outreach activities at TTU, is the Integrated STEM Initiative on the South Plains [3, http:// www.depts.ttu.edu/stem/isisp.php]. This NSF program provides funding for the integration of outreach programs and stimulates changes in the campus culture related to STEM education and outreach. It may be argued that the hiring of an outreach mathematician provided the catalyst and leadership for a more cohesive campus-wide approach to outreach grant writing. As a result, over the last several years more than \$12 million in funding has significantly affected STEM education and outreach projects at TTU. Somewhat ironically, in light of increased funding, a new challenge has been to maintain a focus on the original departmental expectations of the outreach position. Dwyer has had to curtail his role in grass roots activities in order to administer existing projects and coordinate new collaborative programs.

The topic of publication can be a challenge for one in a nontraditional academic role such as an outreach mathematician. The demands of administering outreach must be reconciled with the logistics of producing outreach scholarship. For example, one must address the use of human subjects, Institutional Review Board (IRB) approval, and access to K-12 populations, issues often foreign to traditional mathematics research. There are also both a shortage of appropriate publication outlets and reservations from colleagues concerning the quality of scholarship that results from outreach activities.

A significant development in support of outreach at TTU occurred in 2012 when the university adopted a revised tenure and promotion policy that recognized outreach and community engagement as part of a faculty member's contributions to teaching, research, or service. Even if a discussion of outreach and community engagement does not rise to the university level, a mathematics department should consider amending the reward and promotion structure to take into account the nontraditional role of an outreach mathematician. The mathematics department at TTU has recently adopted such a clause in its promotion and tenure documents. For the record, Dwyer obtained tenure and eventually promotion to full professor, the first such case in his department.

\section{Institutional Perspective}

From the viewpoint of Schovanec as chair and then dean, there are new opportunities, rewards, and frustrations related to outreach mathematics. As chair of a traditional research department at a large state university, he found enthusiasm and appreciation for mathematics outreach at the higher levels of university administration not always commensurate with departmental support.

The goodwill and publicity derived from outreach activities translated into enhanced financial support of the department and a perception of departmental vitality. The Texas Senate recognized the M\&S department when it was awarded the Texas Association of Partners in Education Award, in large part due to activities initiated by Dwyer and his colleagues in the department. Publicity events for major grants garnered significant attention and increased the visibility of the M\&S department at local and regional levels. Furthermore, collaborations with local school districts and regional colleges and alliances with teachers enhanced student recruitment and presented greater opportunities for research and funding.

Since Dwyer arrived at TTU, he has played a critical role in the growing culture of outreach and engagement that now receives greater institutional recognition. Most recently Dwyer has been featured as an Integrated Scholar [4], a distinction that TTU has enlisted to recognize contributions to teaching, research, and service, where outreach is recognized as a component of all three areas. In 2006, TTU was the first Texas university to be included in the Community Engagement 


\section{$\bigwedge \begin{array}{r}\text { American Institute } \\ \text { of Mathematics }\end{array}$

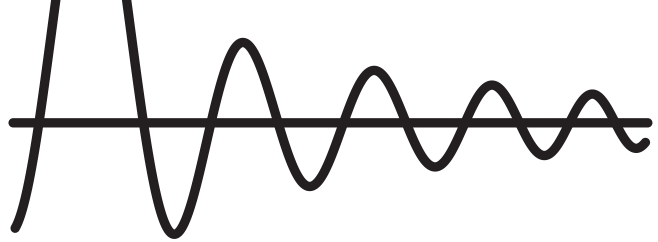

AIM, the American Institute of Mathematics, sponsors week-long activities in all areas of the mathematical sciences with an emphasis on focused collaborative research.

\section{Call for Proposals}

\section{Workshop Program}

AIM invites proposals for its focused workshop program. AIM's workshops are distinguished by their specific mathematical goals. This may involve making progress on a significant unsolved problem or examining the convergence of two distinct areas of mathematics. Workshops are small in size, up to 28 people, to allow for close collaboration among the participants.

\section{SQuaREs Program}

AIM also invites proposals for a new program called SQuaREs, Structured Quartet Research Ensembles. More long-term in nature, this program brings together groups of four to six researchers for a week of focused work on a specific research problem in consecutive years.

More details are available at:

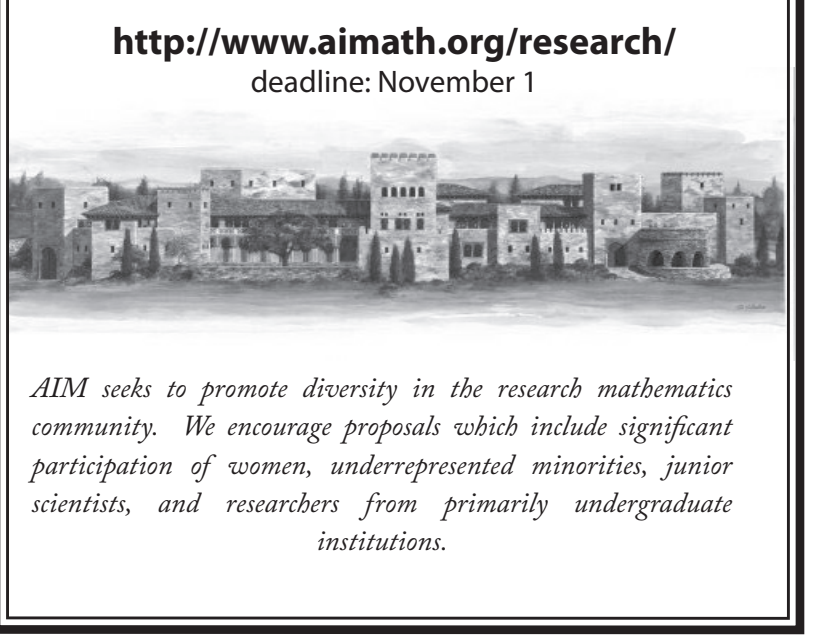

Classification of the Carnegie Foundation for the Advancement of Teaching; it is regularly recognized in the president's Higher Education Community Service Honor Roll. This distinction is partially based on data reflective of TTU's strategic priority to expand community engagement and evidence of extensive faculty-led community collaborations. Since 2009, TTU has annually assessed the institution's community engagement activity utilizing Michigan State's Outreach and Engagement Measurement Instrument (OEMI). Texas Tech has been represented in national and international conversations on institutional mission and assessment of community engagement.

Another promising development is the creation of a TTU university-wide multidisciplinary STEM Center for Outreach, Research, and Education. The center, for which Dwyer serves as director, is supported by six colleges at TTU. This university-wide participation in the center in some ways reflects both authors' vision for the recognition and institutionalization of outreach at TTU.

\section{References}

[1.] J. B. CONWAY, Reflections of a department head on outreach mathematics, Notices of the AMS 48 (10), (2001), 1169-1172.

[2.] J. F. DWYER, Reflections of an outreach mathematician, Notices of the AMS 48 (10), (2001), 1173-1175.

[3.] Integrated STEM Initiative on the South Plains (ISISP), NSF award No. 0930257, September 1, 2009-August 31, 2014.

[4.] Вов Sмiтн, Texas Tech Integrated Scholars, All Things Texas Tech, 3(2), (2011), http://www. depts . ttu.edu/provost/attt/ 